\title{
Large Angle Beamstrahlung as a Beam-beam Monitoring Tool
}

\author{
G. Bonvicini \\ Wayne State University, Detroit MI 48201 \\ J. Welch \\ Cornell University, Ithaca NY 14853
}

September 8, 2018

\section{Introduction}

Luminosity in high energy physics colliders is now more important than ever before. A comparison of 'Livingston' charts, figure 11, shows the machine luminosity increasing at a rate faster than the increase in machine energy. Now and in the forseeable future extremely high luminosity is needed to produce meaningful quantities of rare events (factories) and to cope with the $1 / s$ dependence of the production cross section of high mass states.

Beam alignment complexities with two independent rings, dynamic beta effects, disruption, crossing angle collisions and beamstrahlung are just some of the new phenomena to be dealt with in modern machines running at peak luminosity. Additionally, high luminosity machines must operate near design luminosities in order to be useful, so there is a strong motivation for understanding, controlling, and possibly taking advantage of some these new high luminosity phenomena that occur at the interaction point.

Luminosity for two gaussian beams colliding head-on with equal beam sizes is,

$$
L=\frac{f N_{1} N_{2}}{4 \pi \sigma_{x} \sigma_{y}}
$$

where $f$ is the bunch collision frequency, $N_{1(2)}$ is number of particles per bunch in beam 1(2), and $\sigma_{x(y)}$ is the horizontal(vertical) beam size. Beams are usually quite flat, $\left(r=\sigma_{y} / \sigma_{x}<<1\right)$, with $\sigma_{y}$ being typically a few 
microns or less. The two beam axes must be continously aligned to intersect to much better than $\sigma_{y}$ or luminosity is lost.

For high luminosity conditions, the charge per unit area is made so large that the interaction of one beam with the electromagnetic fields of the other beam can cause poor lifetime, beam blow-up, and other types of instabilities.

One effect of the interaction is to cause the beam size to change significantly over a distance comparable to the length of the bunch. This is called disruption. Since changing the beam size also changes the strength of the interaction, disruption is a very complicated, non-linear phenomenon.

If the beam-beam deflections are significant compared with the average angular spread of particles in the bunch, then the target beam acts like a short high gradient magnet and, in a storage ring, distorts the beta functions all around the ring. This effect is called the dynamic beta effect and has been observed directly at CESR [1]. It is also a highly non-linear effect because the distortion seen by small amplitude particles is largest.

In this paper we propose a method, which has never been used before, to monitor the beam-beam collision conditions. The strength of this method, besides its low cost, versatility, and strong signals well separated in angle from machine backgrounds, is the direct visualization of many aspects of the beam-beam interaction.

As in many other areas of physics research, visualization of a complex phenomenon is at a premium. In this case, we believe a beamstrahlung detector looking at the interaction point might help in faster and better diagnosis of misalignments and incongruities of beam sizes during collision. It might also shed some light on many of the of the dynamical effects which limit the maximum tuneshift parameter and hence the luminosity for a given current. Considering the difficulty in getting a machine into top performance and keeping it there, an instrument, such as we propose, which can directly observe the beam-beam interaction could prove to be quite valuable.

We predict that, if the first such monitor is successfully operated, it will be adopted by the three $e^{+} e^{-}$B-factories (CESR, PEP II, and KEK B), and by the $\phi$-factory in Frascati, and rapidly become the primary beam-beam monitor at all facilities, just as a similar device became primary beam-beam monitor at the SLC. The use of such a monitor by HERA (proton side only) is also a possibility.

We start by discussing the physics of beamstrahlung and what might be expected at the various high luminosity factories and the SLC. Then we go into some detail about 'short magnet' radiation and what it can tell about the collision. Detector design, backgrounds, and possible implementation 

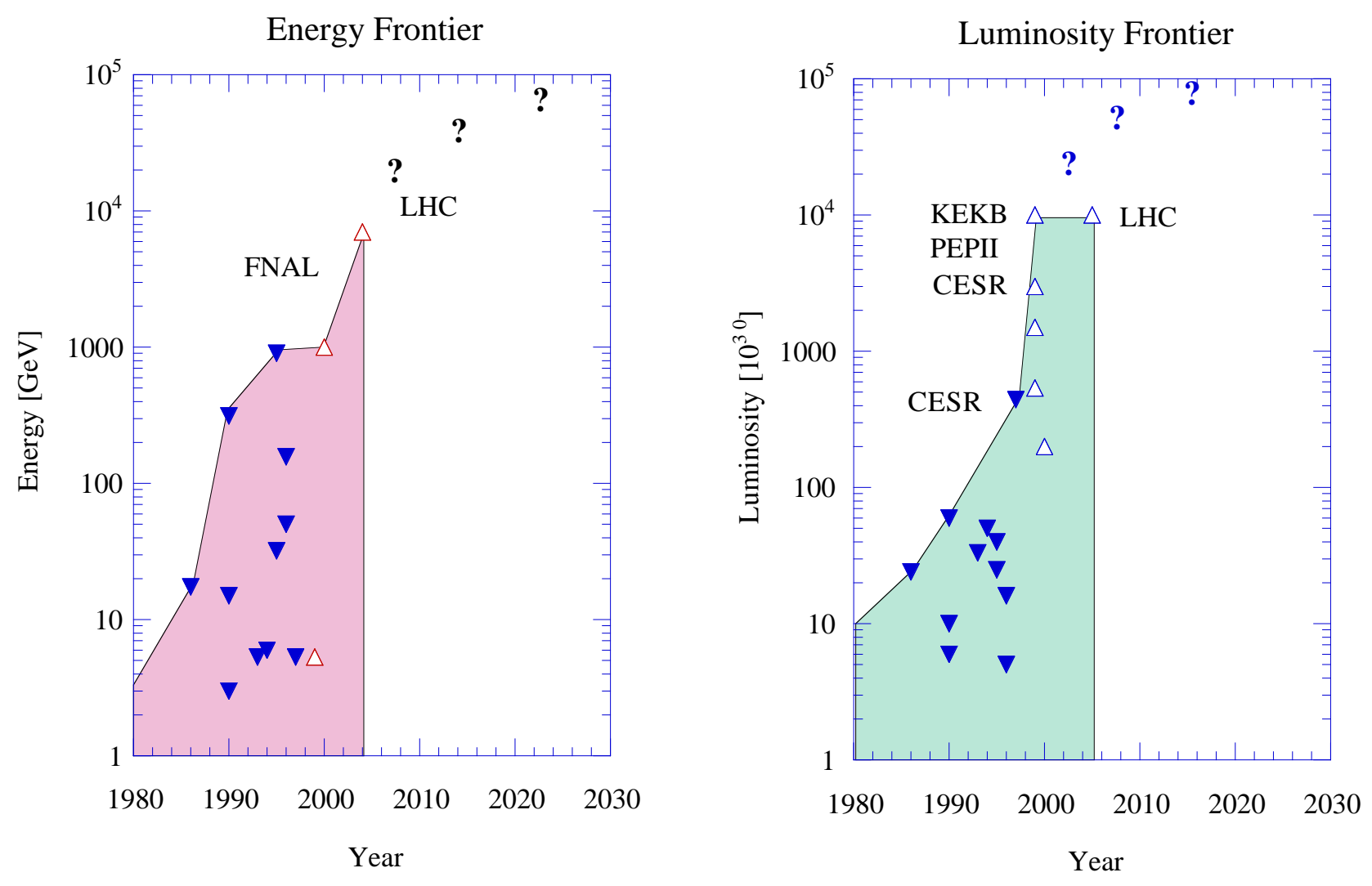

Figure 1: Solid point represent actual obtained peak luminosities (right plot) and energies (left plot) in selected high energy physics accelerators. Open points represent planned values for machines under construction. 
at CESR are then discussed. Finally we address some 'frequently asked questions' concerning beamstrahlung.

\section{Beamstrahlung}

Beamstrahlung occurs when particles of the first beam, or radiating beam, are deflected by the electromagnetic field of the second (target) beam and emit synchrotron radiation. Beamstrahlung was observed first at the SLC [2].

As long as the radiated energy is small compared to the beam energy, most but not all the properties of beamstrahlung can be derived using the formulae in Jackson's "Classical Electrodynamics" [3], convoluted over the space-time distribution of charges, and applying usual ultrarelativistic approximations. The important paper [4] calculates a number of low-energy beamstrahlung properties, which are very relevant to this paper.

Under the conditions of Eq. 1, assuming beams of equal mass and energy, the beamstrahlung power $W_{1}$ is

$$
\begin{aligned}
W_{1} & =f U_{1} \\
& =g(r) r_{e}^{3} m c^{2} \gamma^{2} \frac{f N_{1} N_{2}^{2}}{\sigma_{x} \sigma_{y} \sigma_{z}} \\
& =4 \pi g(r) r_{e}^{3} m c^{2} \gamma^{2} \frac{L N_{2}}{\sigma_{z}},
\end{aligned}
$$

where $U_{1}$ is the energy radiated per collision [1]. Here $r_{e}$ is the classical radius of the beam particles, $m$ their mass, and $\gamma=E / m$ the relativistic factor. $g(r)$ is a dimensionless factor obtained in the integration over spacetime, equal to 4

$$
g(r)=\frac{64 \sqrt{\pi} r}{3 \sqrt{3 r^{4}-10 r^{2}+3}} \arctan \left(\frac{\sqrt{3 r^{4}-10 r^{2}+3}}{3 r^{2}+8 r+3}\right) .
$$

It is maximal for round beams $(r=1)$, at $2.735 \ldots$, and for flat beams $(r$ small) it can be approximated as follows

$$
g(r) \sim 11.4 r
$$

In the flat beam limit, $\sigma_{y}$ cancels in Eq. 3 and the dependence of $W$ on beam parameters becomes

$$
W \propto \frac{\gamma^{2} N_{1} N_{2}^{2}}{\sigma_{z} \sigma_{x}^{2}} .
$$


Table 1: Beamstrahlung parameters derived for high luminosity factories and SLC.

\begin{tabular}{|l|l|r|r|r|r|r|}
\hline & Machine & SLC & CESR 1997 & PEP & KEK & Frascati \\
\hline$L_{\max }$ & {$\left[10^{33} \mathrm{~cm}^{-2} \mathrm{~s}^{-1}\right]$} & 0.0008 & 0.44 & 3. & 10. & 0.13 \\
$r$ & & 0.3 & 0.022 & 0.04 & 0.025 & 0.01 \\
$\sigma_{z}$ & {$[\mathrm{~mm}]$} & 0.8 & 18 & 10 & 4 & 30 \\
$E_{1}$ & {$[\mathrm{GeV}]$} & 50 & 5.3 & 3.1 & 3.5 & 0.51 \\
$E_{2}$ & {$[\mathrm{GeV}]$} & - & - & 9 & 8 & - \\
$N_{1}$ & {$\left[10^{10}\right]$} & 3.5 & 14 & 5.9 & 3.2 & 9 \\
$N_{2}$ & {$\left[10^{10}\right]$} & - & - & 2.7 & 1.3 & - \\
$B_{\sigma 1}$ & {$[T]$} & 200 & 0.14 & 0.17 & 0.43 & 0.01 \\
$B_{\sigma 2}$ & {$[T]$} & - & - & 0.33 & 1.0 & - \\
$W_{1}$ & {$[\mathrm{~W}]$} & 12 & 1.6 & 2.3 & 7.5 & 0.0008 \\
$W_{2}$ & {$[W]$} & - & - & 38 & 95 & - \\
$\theta_{o}$ & {$[\mathrm{mrad}]$} & 27 & 6 & 7 & 12 & 5 \\
$\nu_{1}$ & {$\left[10^{10} s^{-1}\right]$} & $7 \times 10^{-7}$ & 0.34 & 2.0 & 0.32 & 4.6 \\
$\nu_{2}$ & {$\left[10^{10} s^{-1}\right]$} & - & - & 1.2 & 0.15 & - \\
\hline
\end{tabular}

All of these parameters are well known by other means. There is little information in the total emitted power, all of the non-trivial information is in the asymmetries described below. On the other hand, total power becomes a reliably predicted quantity that we use as a constant in the following calculations.

Table 1 summarizes the beam parameters at various facilities, according to Ref. [5] and their beamstrahlung power at design luminosity. It is surprising to discover that beamstrahlung yields at the SLC, PEP-II and KEK are roughly the same, with the higher luminosity compensating for the lower energy and longer beams. Two of the three B-factories feature beams crossing at an horizontal angle, and two feature unequal energy beams. The crossing angle $\alpha_{x}$ is respectively 2 and $10 \mathrm{mrad}$ at CESR and KEK, and results in changes in the total radiated power of $O\left(\alpha_{x}^{2}\right)$. The crossing angle can be neglected except for a trivial distortion of the small angle distributions.

The effect of unequal beams on Eqs. 3 is readily derived with a simple substitution. $\gamma$ is the relativistic factor of the radiating beam, and $\sigma_{z}$ the length of the target beam, and all quantities are defined in the laboratory frame. With these substitutions, Eqs. 3 are valid for beams of unequal 
energies and beam lengths, such as at PEP-II or KEK, in the limit of rigid beams.

Current CESR conditions have to be considered the benchmark. Table 1 lists the total radiated power $W$, as well as other meaningful quantities. At present day CESR, one obtains a total beamstrahlung power of $2.4 \mathrm{~W}$. At PEP-II, the radiated beamstrahlung power is respectively 4 and $80 \mathrm{~W}$ for the low and high energy beams.

Beamstrahlung has been the subject of many theoretical calculations, which are not relevant to this paper, and is a crucial issue for future linear colliders. At the SLC it was detected and monitored primarily to monitor the collision of two beams coming from different beamlines, and therefore having different transverse dimensions and even orientations in the transverse plane. There were seven degrees of freedom in the transverse plane at the SLC (the four beam dimensions, the angle between the two major axes of the transverse ellipses, and the two coordinates of the beam-beam offset). We notice that the situation at asymmetric B-factories is quite similar to the SLC - two beams totally independent of each other. It is at those machines that the device described here will be most useful. The situation at CESR is somewhat more constrained by the single-ring machine.

The North and South beamstrahlung detectors described in Refs. [2] and [6] lasted 6 and 8 years at the SLC without particular problems, delivering instant and primary information on beam-beam overlap to the operators, via a monitor placed directly above the operator's desk. During their lifetime they absorbed close to $100 \mathrm{GRad} /$ year and were designed to minimize visible radiation backgrounds, a fact relevant to this paper.

The SLC monitors worked because the effective magnetic field accompanying the beams, at a typical distance of one standard deviation from the beam center, is

$$
B_{\sigma} \sim \frac{2 N_{2} r_{e} m c}{e \sigma_{z} \sigma_{x}}
$$

which is now of order $100 \mathrm{~T}$ (and was of order $10 \mathrm{~T}$ when beamstrahlung was first observed [2]).

10 Tesla far exceeds all other magnetic fields along the beam line. Thus the beamstrahlung critical energy was much higher than the beam line synchrotron radiation, and although the synchrotron radiation power was one million times higher at the time of first observation, a detector with favorable signal/noise could be built.

Table 1 shows that $B_{\sigma}$ is quite low at today's B-factories, and comparable or lower than the beam line magnetic fields. A signal in this case is extracted 


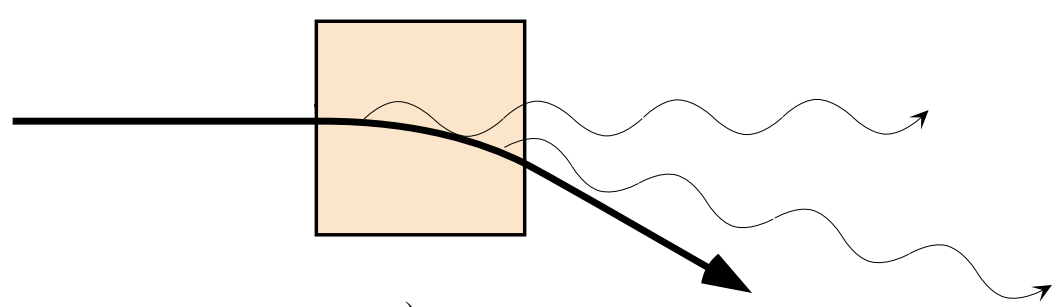

a)

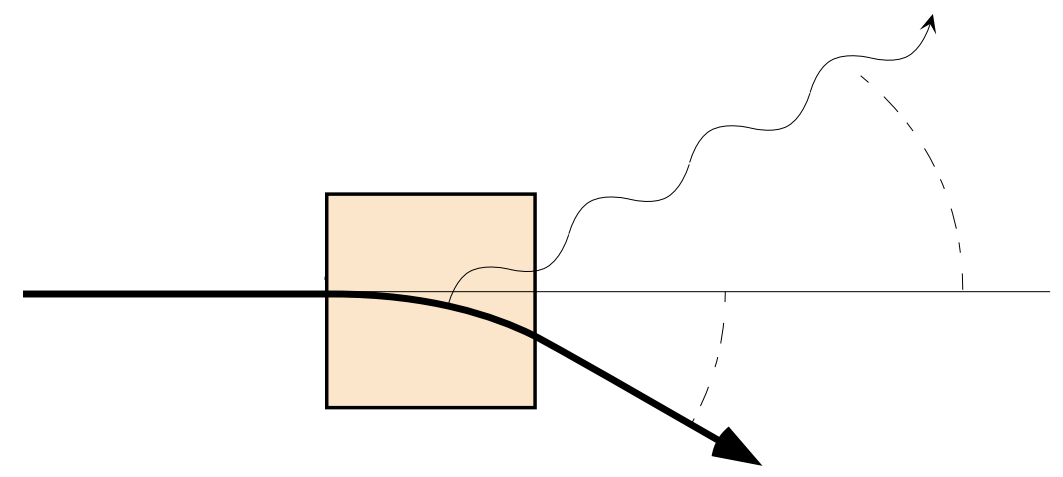

b)

Figure 2: Searchlight approximation: the observation angle is smaller than the bending angle. b) Short magnet approximation: the observation angle is larger than the bending angle.

by noticing that the beam, considered as a magnet, is much shorter than the other magnets. Thus the Fourier transform produces a drastically different radiation spectrum.

"Short magnet" (SM) radiation replaces the usual synchrotron radiation spectrum when the observation angle (Fig. 2) is much larger than the deflection angle. SM extra terms contribute a fraction of the power of order $1 / \gamma^{2}$ of the total power (that is, they integrate to a quantity that has no power dependence on $\gamma$ ), and therefore are perfectly consistent with the "searchlight" approximations for synchrotron radiation [3]. The use of SM radiation as a beam monitor was first suggested in Ref. 海. A practical detector was suggested first at the SLC in Ref. [8]. 


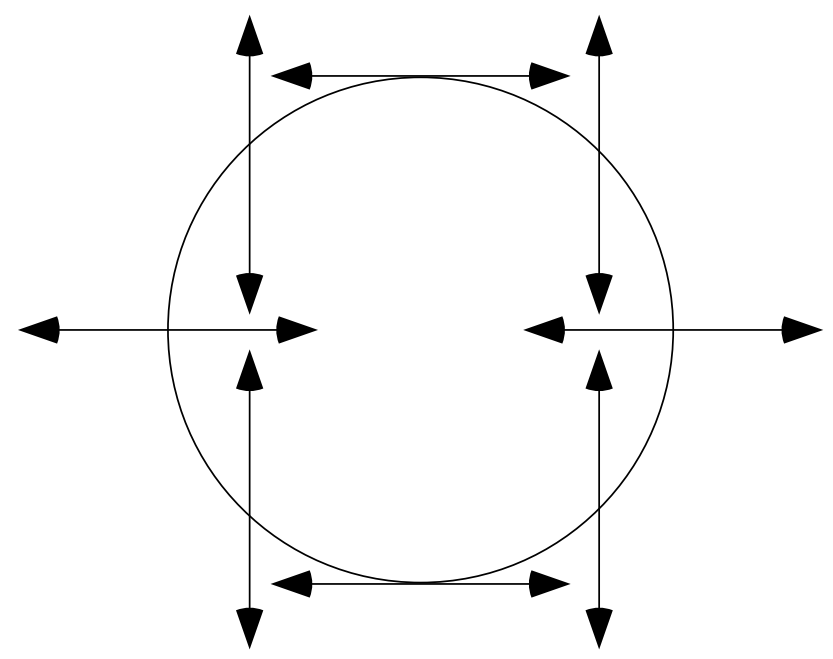

Figure 3: Eightfold polarization pattern for a particle bent in the horizontal direction.

\section{Properties of Short Magnet Radiation}

The properties of SM radiation were first investigated by Coisson [9]. SM radiation was first observed in Ref. [10]. In regions where SM radiation dominates, such as at large angle $(\gamma \theta>>1)$, which are the only ones relevant to this paper, three properties are of interest:

- The large angle power is proportional to $1 / \gamma^{2}$ (or a fraction of $1 / \gamma^{4}$ of the total power). One of the main points of this paper is to show that a possible, but somewhat marginal, signal at the SLC [8] becomes large at the lower energy factories.

- the radiation spectrum is gaussian, and the cutoff frequency independent of beam energy. The spectrum extends far beyond the spectrum calculated in a standard way.

- radiation is linearly polarized parallel or perpendicular to the acceleration, with an eight-fold symmetry in azimuth. Fig. 3 shows the polarization pattern for a vertical bend. 
The large-angle power emitted as a function of angle and frequency is expressed as [4]

$$
I(\theta, \phi, \omega)=\frac{3 \sigma_{z} W_{1}}{4 \pi \sqrt{\pi} c} \frac{1}{\gamma^{4} \theta^{3}} \exp \left(\frac{-\sigma_{z}^{2} \theta^{4} \omega^{2}}{16 c^{2}}\right) .
$$

For reasons that will become clear in the next Section, we compute the power in the visible $\left(3 \times 10^{15}<\omega<6 \times 10^{15} s^{-1}\right)$, that falls within $6 \%$ of the azimuth, and one mrad upward of the optimal angle discussed below (from 6 to $7 \mathrm{mrad}$ for CESR, from 8 to $9 \mathrm{mrad}$ for PEP-II). This is the power readily available for a simple beamstrahlung monitor looking at the beam-beam interaction by means of a few small viewports drilled through the beam pipe. At the optimal angle the exponential factor in Eq. 6 is equal to -2 for photons at the lower $\omega$ limit. For the purposes of this integration the frequency upper limit can be taken to infinity, the error function at -2 is equal to 0.025 and the simple final result is

$$
W_{\text {exp }}=\frac{0.0225 W_{1}}{\gamma^{4}}\left(\frac{1}{\theta_{\min }^{4}}-\frac{1}{\theta_{\max }^{4}}\right) .
$$

The total beamstrahlung power in the narrow phase space region defined above is $0.8 \times 10^{-9}$ of the total power for CESR. When divided by the average photon energy $(2 \mathrm{eV})$ one gets the number of photons per second $\nu_{1}$, listed in Table 1. Current CESR conditions will deliver some 3.5 billion visible photons per second in the region under consideration, or about 1000 photons per beam-beam collision. Clearly this method will give plenty of signal even when the luminosity is one or two orders of magnitude lower than design.

In the case of beam-beam offsets, and general beam-beam diagnostics, things can become quite complex. A good treatise of beamstrahlung patterns versus beam conditions can be found in Gero's thesis [11. A desired result for a particular beam-beam configuration can always be obtained with a straightforward numerical integration over space-time. A detailed treatment of all possible pathologies goes far beyond the scope of a short paper, so we will limit ourselves to a few comments, plus the concepts outlined in the last Section to give an idea of the power of the method.

A beam-beam offset will necessarily generate a dipole moment, which will be reflected in a non-zero polarization of the emitted light, as well as a change in the total light yield. We recall only formulae for the important case of flat beams, experiencing an offset along $y$ [4]. Dividing the power 
emitted into a component parallel to the offset and perpendicular to the offset, the result is

$$
\begin{aligned}
I_{\perp}(\theta, \phi, \omega) & =I(\theta, \phi, \omega) \frac{G_{\perp} \cos ^{2}(2 \phi)+G_{\|} \sin ^{2}(2 \phi)}{G} \\
I_{\|}(\theta, \phi, \omega) & =I(\theta, \phi, \omega) \frac{G_{\perp} \sin ^{2}(2 \phi)+G_{\|} \cos ^{2}(2 \phi)}{G} .
\end{aligned}
$$

The $G$ form factors are defined as

$$
G_{\perp}+G_{\|}=G(r, v), \quad G(r, 0)=g(r) / 2 .
$$

The variable $v$ represents the offset in units of $\sqrt{2} \sigma_{y}$. The polarization asymmetry is defined as

$$
A=\frac{G_{\perp}-G_{\|}}{G(r, v)} .
$$

Fig. 1 shows the $G$ form factors as a function of the offset $v$. The camelback shape is a classical feature of beamstrahlung, due to the radiating beam sampling a region of higher field, and has been used in the past to distinguish beamstrahlung from the background [2].

A lot of information about beamstrahlung patterns can be summarized as follows: the radiation will have the same multipole pattern as the beams have in the transverse plane. If beams are centred and have the same axis, but have different aspect ratios, there will be also a quadrupole pattern. A sextupole moment will develop if one of the beams is rotated with respect to the other.

\section{Design of a Short Magnet Detector.}

The detector must consist of one or more transparent viewports in the beam pipe, at a large enough angle so that synchrotron visible light at zero degrees can be efficiently masked. The photons are then separated by polarization and color and counted.

In the harsh environment surrounding a beamline photomultipliers, with a bialkali photocathode, are known to work well [6]. Thus the frequency acceptance is fixed by the two frequencies where the bialkali efficiency is at half-max $\left(3 \times 10^{15}<\omega<6 \times 10^{15}\right)$. The lower limit is loosely called "red" light and the upper limit "blue" light. 


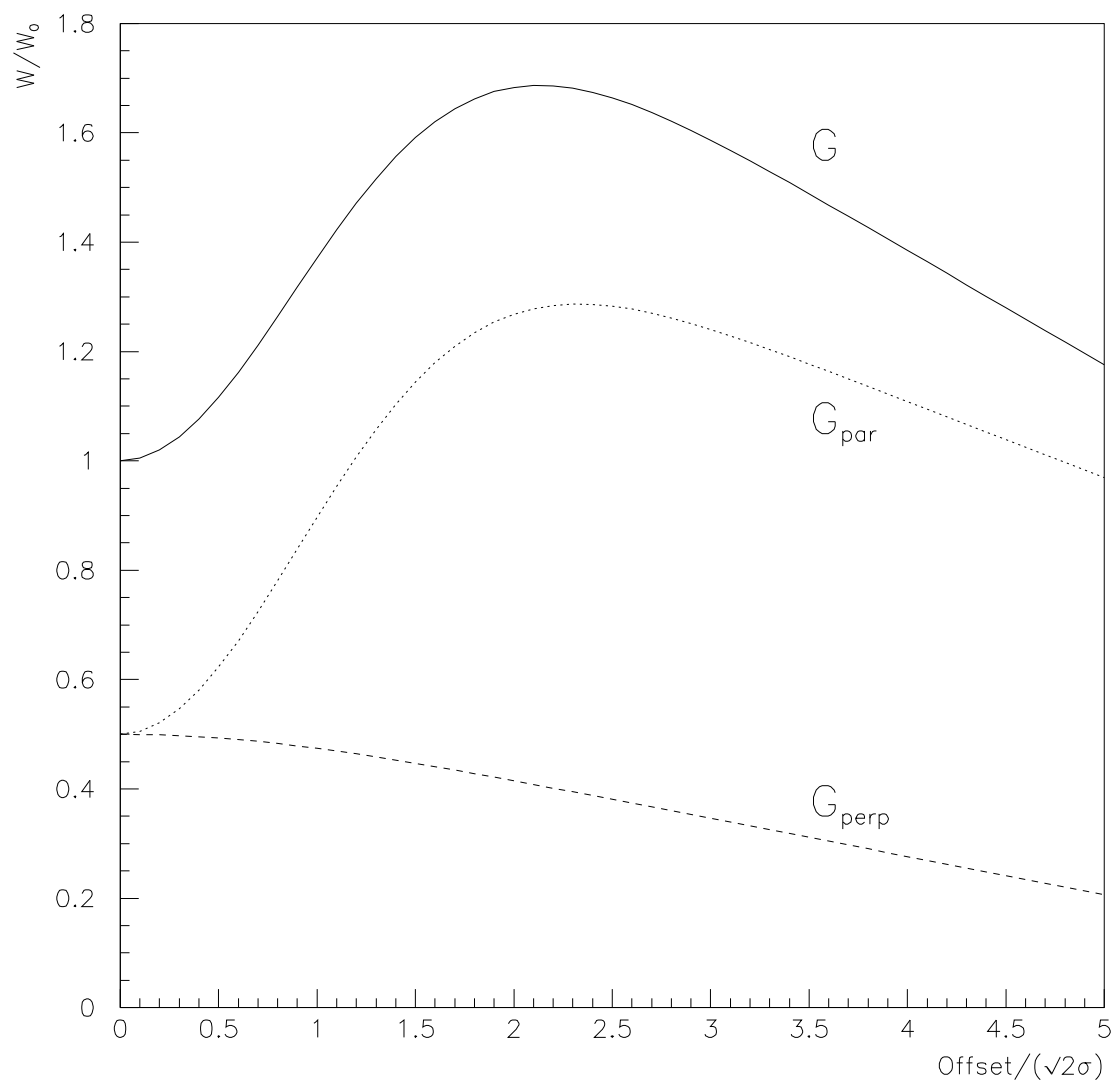

Figure 4: The total power and the components polarized respectively parallel and perpendicular to the bending plane, as a function of the normalized offset. 
We add an "optimal" condition for detection, that is that the exponent in Eq. 6 for red light be equal to two, ie

$$
\theta_{o}=2^{5 / 4} \sqrt{\frac{c}{\omega \sigma_{z}}}=\frac{24 \mathrm{mrad}}{\sqrt{\sigma_{z}(m m)}}
$$

The reason for such a choice is that all backgrounds (Cherenkov radiation, direct synchrotron radiation, fluorescence in materials both in the beam pipe and in the detector) are constant within $60 \%$ across the visible spectrum. By choosing red, and setting the detector at an angle where the blue light is suppressed, we establish that a measure of blue light measures the backgrounds. In other words,

$$
\begin{array}{r}
W_{\text {red }}=e^{-2} W_{\text {signal }}+W_{\text {background }} \\
W_{\text {blue }}=e^{-8} W_{\text {signal }}+W_{\text {background }}
\end{array}
$$

The photon counter would consist of a prism splitting the red and blue components. The blue light goes to its own photomultiplier. The red light is separated in the horizontal and vertical polarization component. Each then goes into a photomultiplier. This background subtraction scheme would improve the sensitivity to a beamstrahlung signal by two orders of magnitude. The exponential factor in Eq. I scales with $\omega \theta^{2}$. If background conditions demand a larger angle, the trick can still be used by using a photocathode sensitive in the near infrared.

Timing information could be had with $0.67 \mathrm{GHz}$ FADCs connected to the PMTs, for the purpose of timing out sources of backgrounds not coming from the interaction region. Timing would improve the sensitivity to a beamstrahlung signal, in the presence of opposite beam backgrounds, by one order of magnitude.

Ideally, a viewport should be located at a fixed grid in azimuth, but avoiding the eightfold pattern of Fig. 3 (every 45 degrees the same information is replicated). Three viewports are needed to fully disentangle the information, and a viewport every 30 degrees is a possibility. In practice, it is best to stay out of the synchrotron radiation sweep (the $x$-axis in Fig. 3 ), to avoid premature ageing of the window and quadrupole synchrotron radiation, and monitor six other locations, for example at 30,60 and 90 degrees, and their opposite locations in azimuth. The radiation pattern is symmetric under 180 degrees rotations in azimuth, and half the monitors are redundant for perfect beam alignment. The redundancy can be used 
to correct beam misalignments. Twelve data would be obtained for seven possible degrees of freedom.

The cons of this proposal are, obviously, beam pipe surgery, and increased heat loads and RF leaks at the viewport location. The latter two can be minimized by drilling several smaller holes per viewport. The heat load and $\mathrm{RF}$ leaks scale like $1 / b^{4}, b$ being the hole radius, thus replacing one hole with three would suppress the leaks by an order of magnitude.

\section{Application at CESR}

A beamstrahlung monitor is being considered for installation in the CESR storage ring to aid in understanding beam-beam effects that occur at high luminosity. CESR has both large dynamic beta effects as well as sizable disruption.

Horizontal and vertical disruption parameters are defined as the bunch length divided by the nominal thin lens focal length in the plane of interest. For gaussian bunches they work out to

$$
D_{h / v}=4 \pi \xi_{h / v} / \sigma_{z}
$$

In CESR they are often as high as 0.5 or more, which indicates that there is substantial change in the transverse beam size during collisions.

The dynamic beta effect is a distortion of beta functions seen by low amplitude particles due to the beam-beam interaction. It is especially significant at high tune shift parameter and when the tune is near a half integer. For example theoretical values for $\beta_{h}^{*} / \beta_{h 0}^{*}<.37$ for a horizontal tuneshift parameter of 0.032 and horizontal betatron number of 0.526 [12].

These types of nonlinear beam-beam distortions affect lowest amplitude particles the most; beam tails are not affected as much. But the low amplitude particles are just the ones that contribute the greatest amount to the overall luminosity, so it would seem advisable to understand their dynamics as thoroughly as possible.

In principle synchrotron light monitors could resolve changes in the average beam profile and detect how they are modified by the collision process. But the analysis of such measurements would be difficult because they are made far from the interaction point and depend on the highly distorted beta functions (at least for low amplitude particles) as well as the beam distribution. Another technique that might address these types of beam-beam effects is to shake one beam and measure the response of the other [13]. 
However while such measurements can provide zeroth order and first order estimates for the beam density, there is no known method for interpreting higher moments.

Given the luminosity as measured by the detector, and the beam currents, beamstrahlung measurements provide direct information about the average vertical beam distribution during the collision. Like the other methods mentioned above, interpretation is not obvious. By scanning the vertical offset of the beams one should be able to see peaks in the beamstrahlung radiation power, and a central minimum whose heights and depths depend on the dynamically changing beam size. As mentioned earlier, polarization data from the beamstrahlung might provide information about moments of the beam distribution, particularly if there is a mismatch between the two beams. These signals could be encorporated in feedback loops to aid in tuning and improve the stability operation.

A proposed location for the beamstrahlung detector would be about 5 $\mathrm{m}$ from the interaction point. Here there is essentially no hard sychrotron radiation from the IR quadrupoles. About $2 \mathrm{~W} / \mathrm{cm}$ of soft bend dipole radiation is deposited in this region with a critical energy of $2 \mathrm{keV}$. This location is also favored by a small beam stay-clear in both planes which allows more penetration into the vacuum chamber with mirrors. The average angle for beamstrahlung radiation striking the mirror would be $6.2 \mathrm{mr}$. A mirror with a $1 \mathrm{~cm}$ penetration and 1 in diameter would intercept a solid angle of $2 \times 10^{-4} \mathrm{sr}$.

Collimation can be arranged so that the only background synchrotron radiation comes from the opposite side of the interaction point, which is about $10 \mathrm{~m}$ away, and such radiation is scattered once off the vacuum chamber.

\section{Backgrounds.}

We preliminarly study the CESR case, where the available beamstrahlung power at the viewports is about $2 \mathrm{nW}$. As shown in Section 5, the power deposited in the region around the detector is about $2 \mathrm{~W} / \mathrm{cm}$ along the main synchrotron sweep. Therefore a background reduction factor of order $10^{-9}$ will suffice. The backgrounds off the main sweep are perhaps three orders of magnitude less. Three types of backgrounds are distinguished: radiation, visible and irreducible backgrounds.

Radiation backgrounds are caused by radiation generating fluorescence or Cherenkov light in the beamstrahlung detector itself. Experience from 
Refs. [2] and [6] is of guidance. Radiation backgrounds drop very sharply with distance from the beam pipe, thus only the radiation hitting the primary mirror and the primary window needs to be considered. Neither the window nor the mirror are hit by the main sweep, and both could be somewhat recessed inside the beam pipe. The conversion factor for radiation energy into Cherenkov light in glass-like media is about $10^{-4}$ for relativistic particles, and virtually zero for synchrotron radiation (SR) with a critical energy of less than $10 \mathrm{keV}$. The window would be well recessed from the beam pipe, and shielded from beam halo, further reducing the Cherenkov backgrounds by perhaps two orders of magnitude.

The conversion factor for fluorescence varies considerably from material to material [6], but it can be conservatively estimated to be $10^{-6}$ to $10^{-8}$. For metals only, it is strongly dependent on the energy deposition mean depth, as only the atoms at the skin depth can emit visible radiation. The solid angle factor to a small iris could be of order $10^{-5}$ for both window and mirror. By multiplying all the reduction factors together one obtains conservatively a reduction of $10^{-11}$ for Cherenkov light in the window, and $10^{-14}$ for fluorescence. These source of backgrounds should be negligible by several orders of magnitude.

Visible backgrounds (VB) are due to visible light emitted by the inner surface of the beam pipe, either by beam pipe fluorescence, reflection and scattering of visible light (mostly directly produced SR), or a combination of both. At least at CESR, one detector would image the interaction point, and the detector on the other side. Since the last scattering would happen on the opposite detector, a variety of methods can be used to minimize VB. Firstly, the flanges can be made somewhat rough, to insure that reflection is isotropic and not forward peaked. Most important of all, if the detector is truly symmetric in azimuth, individual viewports would be looking at another recessed viewport, if the detector is focussed on the other detector and not on the IP. In all cases the solid angle factor is about $10^{-8}$, and the opposite detector is not hit by the main sweep. Thus a reduction of at least $10^{-11}$ should be possible, and this source of backgrounds is likely negligible.

Irreducible backgrounds are produced by particles in the beam tails, as they get strongly focussed in the final quadrupoles. At CESR, there are perhaps $10^{6}$ particles bent by more than $5 \mathrm{mrad}$ in the vertical direction in the last quadrupole. This kind of background is clearly irreducible, but falling extremely sharply. A detailed calculation is complex, and beyond the scope of this paper. Should it prove to be a problem, moving the detection frequency (and therefore the optimal angle) could prove to be the solution. 


\section{Beamstrahlung Patterns: the FAQs}

In conclusion, we have shown that accurate beam-beam monitoring is easily achievable at all existing or planned $e^{+} e^{-}$particle factories. The preferred method is the monitoring of large angle visible light beamstrahlung, which is produced in abundance (Table 1), and carries information about the beambeam collision conditions, not only limited to luminosity but also beambeam offsets, shape differences, and other transverse mismatches. The total diagnostic handbook goes far beyond the scope of a short paper, but we wish to conclude with the Frequently Asked Questions file about beamstrahlung phenomenology [11]. Hopefully it might be of use sometime in the future.

- Q. How is red light polarization used? Polarization is always set to a minimum by initial steering, for optimal beam-beam overlap. The onset of a non-minimal polarization during a run requires corrective action. Stable beam conditions is obtained at a double local minimum point of minimal polarization and minimal power emitted. Zero polarization is achieved only if the two beams match perfectly in the transverse plane.

- Q. How to do the steering to minimize polarization? If the offset is purely in the $x-(y-)$ axis, the counters at 90 and 270 degrees will show an excess in their respectively horizontal(vertical) polarization counters. Remedial action is steering (say, for $y$ misalignments) up, and if polarization increases, down.

- Q. Can red light give information about optics optimization? Yes. Successfully squeezing the target beam will always result in higher power from the radiating beam, and lower power for the target beam, for optimal overlap. The reverse is also true. As a general rule, in a comparison of equal energy, equally intense beams, the wide beam radiates more than the narrow beam, all else being equal.

- Q. What if the two flat beams have a non-zero angle between the major axes? That will in principle generate a situation where the minimal polarization is non-zero. The beam with the widest $y$ profile (in case of flat beams, the one which has rotated) will radiate the most in the vertical polarization, all else being equal.

- Q. What if the beams have different aspect ratios? The rule of widest beam radiating the most is very far reaching. The widest beam in $y$ 
will radiate the most in $y$, the other beam will radiate the most in $x$, all else being equal.

- Q. What can be learned from measuring the frequency spectrum at large angle? By moving, under stable beam conditions, the signal PMT across the visible spectrum, the beam length can be measured accurately. This is a second reason to place the detector at the optimal angle described above. A frequency scan at a substantially lower angle would not work, because the gaussian factor becomes flat.

\section{References}

[1] D. Cinabro et al., physics/9707001.

[2] G. Bonvicini et al., Phys. Rev. Lett. 62: 2381, 1989.

[3] J. D. Jackson, "Classical Electrodynamics", Chapter 14. The "searchlight" approximation for synchrotron radiation is inserted between Eqs. (14.76) and (14.77).

[4] M. Bassetti et al., IEEE Trans. Nucl. Science 30: 2182, 1983.

[5] Particle Data Book 1996, Phys. Rev. D 54, 1996, pages 129 and 130.

[6] G. Bonvicini et al., Nucl. Instr. and Meth. 277, 297, 1989.

[7] See for example R. Hollebeek, Nucl. Instr. and Meth. 184, 331, 1981.

[8] G. Bonvicini et al., SLAC-PUB-3978.

[9] R. Coisson, Phys. Rev. A 20, 524, 1979.

[10] G. Bossart et al., Nucl. Instr. and Meth. 164, 375, 1979.

[11] E. Gero Ph. D. Thesis, University of Michigan, 1989. See also E. Gero et al., SLAC-PUB-4930.

[12] D.C. Sagan, Dynamic Beta Effect in CESR, http://www.lns.cornell.edu/public/CBN/1994, CBN 94-05.

[13] D.C. Sagan, J.P. Sikora, S.D. Henderson, A Luminosity Monitor Using Coherent Beam-Beam Interaction, http://www.lns.cornell.edu/public/CBN/1997, CBN 97-13. 